\title{
Eletrocardiografia pré-operatória em 474 cães
}

[Pre-operative electrocardiography evaluation in 474 dogs]

\author{
C.F. Carvalho ${ }^{1}$, E.A. Tudury ${ }^{2}$, I.V. Neves ${ }^{3}$, T.H.T. Fernandes ${ }^{3}$, \\ L.P. Gonçalves ${ }^{3}$, R.R.C.L. Salvador ${ }^{3}$ \\ ${ }^{1}$ Médica veterinária autônoma \\ ${ }^{2}$ Departamento de Medicina Veterinária - UFRPE \\ R. Dom Manoel de Medeiros, $\mathrm{s} / \mathrm{n}$ \\ 52171-900 - Recife, PE \\ ${ }^{3}$ Aluno de graduação - UFRPE - Recife, PE
}

\begin{abstract}
RESUMO
Foram realizadas avaliações eletrocardiográficas pré-operatórias em 474 cães, machos e fêmeas, com o objetivo de analisar a frequência de alterações eletrocardiográficas e sua relação com indicação cirúrgica, sexo, idade, raça, peso, escore corporal (obesidade), histórico e sinais físicos de doenças cardíacas. As alterações eletrocardiográficas foram observadas em 220 animais (46\%), sendo mais frequentes nos machos $(\mathrm{n}=116$; $52,7 \%)$. O aumento na duração do QRS foi a alteração mais observada ( $n=54 ; 24,5 \%)$. Sugere-se o exame eletrocardiográfico de rotina na avaliação pré-cirúrgica, independentemente de idade, peso, histórico ou sinais físicos de cardiopatia.
\end{abstract}

Palavras-chave: cão, eletrocardiograma, pré-operatório, arritmia

\begin{abstract}
Pre-operative electrocardiographic evaluations were done in 474 male and female dogs to analyze the frequency of electrocardiographic alterations and its relation with the surgical indication according to age, breed, weight, body score (obesity), and history and physical signs of cardiac diseases. The electrocardiographic alterations occurred in 220 (46\%) animals, being more frequent in males $(n=116$; $52.7 \%)$. The increase in QRS duration was the most observed alteration ( $n=54 ; 24.5 \%)$. The electrocardiographic alterations in the pre-operative are common, which suggests that the performing of electrocardiographic exam in the pre-operative is independent of the age, weight, surgical problem, or signs of cardiopathy on clinical exam.
\end{abstract}

Keywords: dog, electrocardiogram, pre-operative, arrhythmia

\section{INTRODUÇÃO}

As arritmias são definidas como anormalidades de formação, condução, frequência e regularidade do impulso cardíaco, onda e intervalo entre elas (Carr et al., 2002; Ramirez et al., 2003), assumindo o exame eletrocardiográfico, papel fundamental no diagnóstico das arritmias (Rogers et al., 1993). Tudury et al. (2003) relataram a ocorrência de distúrbios do ritmo, frequência e/ou funcionamento cardíaco durante os procedimentos cirúrgicos em cães e gatos. A verificação de arritmia na avaliação pré-anestésica permite a escolha do fármaco isento de potencial arritmogênico, o que torna o exame eletrocardiográfico imprescindível no préoperatório (Rabelo, 2004; Ferreira et al., 2006).

De maneira geral, o eletrocardiograma préoperatório deve ser realizado nos animais em que o exame físico e o histórico sugerem alguma cardiopatia e naqueles com mais de seis anos (Fries, 1993; Futema, 2002). O coração do cão idoso é propenso à fibrilação atrial (Anyukhovsky et al., 2002), e nessa idade a incidência de arritmias assintomáticas e de insuficiência valvar é alta (Fries, 1993).

Recebido em 16 de junho de 2008

Aceito em 10 de março de 2009

E-mail: cecycarvalho@yahoo.com.br 
São várias as doenças cardíacas que levam ao desenvolvimento de arritmias, sendo a endocardiose de valva mitral a cardiopatia mais comum em cães (Abbott, 2002). O eletrocardiograma também pode ser utilizado para averiguar mudanças não específicas como os efeitos de distúrbios metabólicos ou eletrolíticos sobre o miocárdio (Kelly, 2000), pois um coração morfologicamente normal pode apresentar um eletrocardiograma alterado como consequência de doença sistêmica (Lunney e Ettinger, 1997; Goodwin, 2002). Nesse caso, a eletrocardiografia representa uma via rápida $\mathrm{e}$ eficiente para se obter dados consideráveis acerca do estado geral e cardiovascular do paciente (Brourman et al., 1996). Distúrbios eletrocardiográficos na miocardite bacteriana podem ser observados (Edwards, 1987; Sisson et al., 1999), verificando-se, também, complexo ventricular prematuro, taquicardia ventricular e anormalidades no intervalo QT e na onda $\mathrm{T}$ (Tilley, 1992).

A presença de cardiopatia não contraindica a cirurgia, mas é uma consideração importante na decisão de realizá-la e na determinação de um protocolo anestésico ideal (Fries, 1993). As informações obtidas orientam a equipe cirúrgica e o anestesista nos cuidados no ato operatório, a fim de tornar o procedimento o mais seguro possível (Paddleford, 2001). A avaliação préoperatória deve ser feita para todo tipo de cirurgia, visando a estratégias de tratamento e à prevenção de complicações (Rogers et al., 1993).

Pode-se esperar aumento das complicações no transcirúrgico em pacientes com insuficiência cardíaca congestiva, miocardiopatia, bloqueio atrioventricular completo, complexos atriais ou ventriculares prematuros, associados com alterações hemodinâmicas (especialmente com evidência de ascite), edema pulmonar, intolerância ao exercício, dispneia e síncope ou tosse secundário a doença cardíaca (Fries, 1993; Orton, 2002).

Este trabalho teve como objetivo avaliar a frequência e o tipo de anormalidades eletrocardiográficas presentes no exame préoperatório de cães atendidos em hospital veterinário e estudar a sua relação com a presença de enfermidade cirúrgica, histórico e sinais físicos de cardiopatia, obesidade, idade, sexo e peso do animal.

\section{MATERIAL E MÉTODOS}

Foram avaliados 474 cães, 167 machos e 307 fêmeas, encaminhados ao setor de cirurgia do Hospital Veterinário da Universidade Federal Rural de Pernambuco, no período de março de 2005 a dezembro de 2006. Inicialmente foi aplicado questionário direcionado abordando dados referentes às características físicas - raça, idade, peso e sexo - e ao histórico do paciente, visando obter informações a respeito da função cardiovascular e do estado geral, além da indicação cirúrgica, seguido de exames físicos geral e cardiovascular. Os pacientes com alterações na ausculta, frequência e ritmo cardíaco, anormalidades no pulso arterial e sinais de congestão foram considerados como portadores de sinais de cardiopatia ao exame físico.

O eletrocardiograma foi realizado com o equipamento padronizado para sensibilidade de $1 \mathrm{~cm}$ para cada milivolt $(\mathrm{mV})$ e registro na velocidade de $50 \mathrm{~mm} / \mathrm{s}$, nas derivações bipolares e unipolares aumentadas de membros, com o animal posicionado em decúbito lateral direito, utilizando-se o eletrocardiógrafo modelo $\mathrm{C}_{10}{ }^{1}$, com capacidade para registro pelos métodos convencional e computadorizado, tendo sido utilizado o método convencional em 204 animais e o computadorizado em 270.

Foram verificados: frequência e ritmo cardíacos; duração (em milissegundos-ms) da onda $\mathrm{P}$, do complexo QRS e dos intervalos PR e QT; amplitudes $(\mathrm{mV})$ das ondas $\mathrm{P}, \mathrm{R}$ e $\mathrm{T}$; polaridade da onda $\mathrm{T}$; nivelamento do segmento ST e eixo elétrico médio (Goodwin, 2002), cujo laudo foi baseado na avaliação de três a quatro complexos. O eletrocardiograma foi considerado com alteração quando se verificaram anormalidades na formação, condução, frequência e regularidade do impulso cardíaco e na onda e intervalo entre elas (Carr et al., 2002; Ramirez et al., 2003), seguindo a análise e os valores de duração $(\mathrm{ms})$, amplitude $(\mathrm{mV})$ e frequência cardíaca estabelecidos por Tilley (1992) para o método convencional, e por Wolf et al. (2000), para a eletrocardiografia computadorizada.

No estudo da indicação cirúrgica, a população foi dividida em dois grupos: um encaminhado para

${ }^{1}$ Tecnologia Eletrônica Brasileira - São Paulo, Brasil. 
procedimentos de ovariossalpingo-histerectomia e orquiectomia, constituído de cem animais clinicamente saudáveis, e outro, com enfermidades cirúrgicas como neoplasia mamária e fratura de ossos longos, dentre outras, denominadas aqui de cirurgias diversas, constituído de 374 cães. Para este último grupo, foi realizada também avaliação de cada enfermidade cirúrgica para a verificação de uma possível predileção ao desenvolvimento de arritmias.

Foram considerados obesos os cães com acúmulo excessivo de gordura corpórea detectada por meio de inspeção e palpação de acordo com Nelson e Couto (2001). Cães com menos de sete meses não fizeram parte da amostra. Para a variável idade, foi feita uma padronização relacionando-a ao porte do animal, uma vez que a cronologia é diversa para os diferentes portes analisados, de forma que os animais fossem classificados em filhotes, adultos e idosos em três categorias: pequeno porte (até $10 \mathrm{~kg}$ e: de sete a 10 meses, filhote, de 11 meses a oito anos, adulto, e com mais de oito anos, idoso); médio porte $(11$ a $25 \mathrm{~kg}$ e: de sete a 12 meses, filhote, de um ano a sete anos, adulto, e com mais de sete anos, idoso) e grande porte (mais de $25 \mathrm{~kg}$ e: de sete a 18 meses, adulto, e com mais cinco anos, idoso), e analisados separadamente (Grandjean, 2001). A população foi predominantemente adulta para cães de pequeno e médio porte e idosa para cães de grande porte.

Considerando-se que a grande variação na conformação corporal e a raça podem alterar as medidas eletrocardiográficas padronizadas (Tilley, 1992), utilizaram-se, durante a interpretação dos eletrocardiogramas, os padrões de peso: até 9,9kg (193 cães), de 10 a 20kg (186 cães) e acima de $20 \mathrm{~kg}$ (95 cães), conforme sugeriram Wolf et al. (2000), a fim de analisar a atividade elétrica cardíaca de uma maneira correta e adequada.

A análise estatística (teste do qui-quadrado) foi feita relacionando as diversas variáveis: presença de enfermidade cirúrgica, histórico e sinais físicos de doença cardíaca, obesidade, idade, sexo e peso do animal com a presença ou ausência de alterações no eletrocardiograma em dois níveis $(\mathrm{P}<0,05$ e $\mathrm{P}<0,01)$ para a população geral e para as raças mais frequentes (SRD e Poodle). Ademais, foram verificadas quais as alterações eletrocardiográficas mais frequentes na população geral de acordo com o sexo e o estado corporal (obesidade).

\section{RESULTADOS E DISCUSSÃO}

Nesse estudo, 219 (46\%) cães apresentaram alterações, indicando alta frequência de anormalidades. $\mathrm{O}$ percentual verificado por Rabelo (2004) foi ainda maior (65\%). As alterações eletrocardiográficas mais encontradas foram aumento na duração do complexo QRS e aumento na amplitude da onda $\mathrm{T}$ (Tab. 1), semelhante ao resultado observado por Rabelo (2004). Uma vez que o aumento na duração do complexo QRS é indicativo de sobrecarga ventricular esquerda (Tilley, 1992; Kittleson, 1998; Abbott, 2002), é possível que a alta prevalência dessa alteração possa estar ligada ao fato de a degeneração mixomatosa da valva mitral ser a cardiopatia mais comum (Abbott, 2002).

Quanto às enfermidades cirúrgicas avaliadas, a afecção mais frequente (Fig. 1) foi a neoplasia mamária $(18,4 \%)$, seguida de neoplasia em sistemas variados $(15 \%)$ e fratura de ossos longos e pelve $(13,8 \%)$. Não se observou predisposição significativa $(\mathrm{P}>0,05)$ para $\mathrm{o}$ aparecimento de arritmias em uma determinada enfermidade cirúrgica em relação às outras.

Segundo a raça, em ordem decrescente, foram avaliados: 230 cães SRD (48,5\%); 108 Poodles (22,8\%); 25 Cocker Spaniels (5,3\%); 22 Pastores Alemães (4,6\%); 21 Pinschers (4,4\%); 11 Dobermans $(2,3 \%)$, nove Dachshund $(1,9 \%)$, oito Yorkshires $(1,7 \%)$, cinco Boxers $(1,1 \%)$ e outras raças que totalizaram $35(7 \%)$. Como os cães SRD e da raça Poodle juntos representaram mais de $70 \%$ dos animais avaliados, essas raças foram analisadas em separado. Os cães que apresentaram maior percentual de alterações eletrocardiográficas foram: Doberman $(63,6 \%)$, Boxer $(60,0 \%)$, Yorkshire $(50,0 \%)$, SRD $(47,8 \%)$ e Poodle $(46,3 \%)$. A maior percentagem de alterações eletrocardiográficas observadas em Dobermans e Boxers nessa amostra pode ser atribuída à predisposição dessas raças a desenvolverem cardiomiopatia dilatada, aspecto já relatado por Calvert (2002). Isto, entretanto, não pôde ser comprovado neste estudo em virtude da pequena representatividade na amostra. 
$\mathrm{Na}$ população, 374 animais (79\%) foram encaminhados para cirurgias diversas, e $46,8 \%$ destes apresentaram alterações eletrocardiográficas. Em 100 pacientes (21\%) recomendados a gonadectomias, a frequência de alterações eletrocardiográficas também foi elevada, $44 \%$. Na população de cães SRD, constituída por 230 animais, 49,7\% dos 151 encaminhados para cirurgias diversas e 44,3\% dos cães encaminhados para castrações apresentaram alterações. Nos da raça Poodle representados por 108 animais, 47\% dos 100 animais encaminhados para cirurgias diversas e 37,5\% dos oito encaminhados para gonadectomias apresentaram alterações. Desse modo, não houve diferença significativa $(\mathrm{P}>0,05)$ quanto à presença de alterações eletrocardiográficas nas populações analisadas, confirmando os estudos de Roger et al. (1993) de que a elevada taxa de alterações eletrocardiográficas independente da presença de enfermidade cirúrgica.

De acordo com Tilley (1992) e Gompf (2002) os machos são mais propensos a desenvolverem doenças cardíacas. Neste estudo, observou-se maior frequência $\quad(\mathrm{P}<0,05) \quad$ de $\quad$ alterações eletrocardiográficas nos machos, $52,7 \%$, ou 88 animais de uma população de 157 machos. Nas fêmeas, o valor foi de $42,3 \%$ ou 136 animais de uma população de 307 fêmeas. Para os 230 cães $\mathrm{SRD}$, foram verificadas alterações em $55,5 \%$ dos 63 animais do sexo masculino e 44,9\% dos 167 do sexo feminino. Nos Poodles, representados por 108 animais, $56,1 \%$ dos 41 cães machos apresentaram alterações contra 40,3\% das 67 fêmeas. A presença de Sinus arrest foi mais frequente $(\mathrm{P}<0,01)$ nas fêmeas que nos machos, $80 \%$ e $20 \%$, respectivamente (Tab. 1), não sendo observada relevância em relação às demais alterações identificadas.

Na população de cães SRD, 63,9\% dos 36 obesos e $44,8 \%$ dos 194 não obesos apresentaram alterações eletrocardiográficas, isto é, a proporção de obesos com anormalidades foi mais elevada $(\mathrm{P}<0,05)$. Estes resultados confirmam os já obtidos por Jericó et al. (2006), que verificaram frequência de $72,4 \%$ de cães obesos com alterações em uma população de 69 animais, ressaltando, assim, a importância da avaliação eletrocardiográfica pré-operatória nos animais obesos. Na população de Poodles, não se verificaram essas variações entre obesos e não obesos.

Jericó et al. (2006) observaram que as alterações do segmento ST $(50,7 \%)$ foram as anormalidades mais frequentes observadas em cães obesos. No presente estudo, essa percentagem foi de apenas 5,3\%.
Apesar de o aumento na duração do complexo QRS ter sido a alteração mais frequente verificada nos cães obesos $(24,3 \%)$, a única alteração que se mostrou relevante $(\mathrm{P}<0,05)$ nessa população foi $\mathrm{o}$ complexo QRS de baixa voltagem (Fig. 2), reforçando a observação feita por Tilley (1992) de que a obesidade pode interferir na amplitude do complexo QRS. Isto denota a necessidade de uma análise mais criteriosa do complexo QRS em cães obesos.

Nos cães SRD, 64,4\% de 45 cães que tinham histórico de cardiopatia apresentaram alterações eletrocardiográficas, contra $43,8 \%$ de 185 animais que não apresentavam histórico. Comparando com a população geral em que $52,3 \%$ de 132 animais com histórico e 43,9\% de 342 animais sem histórico apresentaram alterações, e com a população de Poodles em que $51,1 \%$ de 47 animais com histórico e $42,6 \%$ de 61 animais sem histórico apresentaram alterações, verificou-se que apenas na população de cães SRD a presença de histórico estava relacionada à presença de alterações eletrocardiográficas $(\mathrm{P}<0,01)$. Estes resultados indicam que as alterações eletrocardiográficas nem sempre estão associadas à presença de cardiopatia (Lunney e Ettinger, 1997; Goodwin, 2002). Ressalta-se, dessa forma, a importância de realizar esse exame também nos animais que não apresentem histórico de cardiopatia.

A presença de alterações eletrocardiográficas não dependeu da presença de sinais, ao exame físico, que indicassem cardiopatia $(\mathrm{P}>0,05)$ (Fig. 3). Com isso, pode-se ressaltar a importância da avaliação eletrocardiográfica pré-operatória nos pacientes em que o exame físico não sugira cardiopatia. Fries (1993) e Futema (2002) indicaram o contrário.

A frequência de alterações eletrocardiográficas dos 193 cães da população geral com até $9,9 \mathrm{~kg}$ foi de $45,1 \%$; para os 186 animais com pesos entre 10 a $20 \mathrm{~kg}$, foi de $47,9 \%$, e para os 95 animais com mais de $20 \mathrm{~kg}, 45,3 \%$. Essas percentagens foram verificadas também nas categorias de peso avaliadas dos cães SRD e Poodle. Devido à não significância $(\mathrm{P}>0,05)$ para a relação peso/frequência de alteração nas três populações, fica evidente a importância do uso do eletrocardiograma, independente do peso.

O potencial que os cães jovens têm de desenvolver arritmias sérias no pós-operatório é real, o que por si só justifica a necessidade de avaliação préoperatória nesses pacientes (Duerr et al., 2007). O fato de não ter sido verificada diferença 
significativa $(\mathrm{P}>0,05)$ quanto à presença de alterações eletrocardiográficas em cães filhotes, adultos e idosos (Fig. 4) reforça a importância do eletrocardiograma no pré-anestésico de cães jovens, diferente do que foi indicado por Futema (2002). O elevado numero de cães jovens com histórico de atropelamento nessa amostra pode ter resultado na alta frequência de alterações, uma vez que o acidente automobilístico predispõe ao desenvolvimento de miocardite traumática e consequente arritmias (Snyder et al., 2001).

Tabela 1. Número e percentagem de alterações eletrocardiográficas verificadas no pré-operatório de 219 cães atendidos no período de 03/2005 a 12/2006, em hospital veterinário*

\begin{tabular}{|c|c|c|c|c|c|c|c|c|c|c|}
\hline \multirow{3}{*}{$\begin{array}{l}\text { Alteração } \\
\text { eletrocardiográfica }\end{array}$} & \multicolumn{2}{|c|}{ Geral } & \multicolumn{4}{|c|}{ Sexo } & \multicolumn{4}{|c|}{ Estado corporal } \\
\hline & \multirow[b]{2}{*}{$\mathrm{n}$} & \multirow[b]{2}{*}{$\%$} & \multicolumn{2}{|c|}{ Macho } & \multicolumn{2}{|c|}{ Fêmea } & \multicolumn{2}{|c|}{ Obeso } & \multicolumn{2}{|c|}{ Não obeso } \\
\hline & & & $\mathrm{n}$ & $\%$ & $\mathrm{n}$ & $\%$ & $\mathrm{n}$ & $\%$ & $\mathrm{n}$ & $\%$ \\
\hline $\begin{array}{l}\text { Aumento na duração } \\
\text { do QRS }\end{array}$ & 71 & 24,3 & 28 & 39,4 & 43 & 60,6 & 15 & 21,1 & 56 & 78,9 \\
\hline $\begin{array}{l}\text { Aumento na amp. de } \\
\mathrm{T}\end{array}$ & 60 & 20,5 & 27 & 45 & 33 & 55 & 11 & 18,3 & 49 & 81,7 \\
\hline $\begin{array}{l}\text { QRS de baixa } \\
\text { voltagem }\end{array}$ & 25 & 8,6 & 7 & 28 & 18 & 72 & 9 & 36 & 16 & 64 \\
\hline $\begin{array}{l}\text { Desvio do eixo para } \\
\text { esquerda }\end{array}$ & 25 & 8,6 & 10 & 40 & 15 & 60 & 5 & 20 & 20 & 80 \\
\hline $\begin{array}{l}\text { Desvio do eixo para } \\
\text { direita }\end{array}$ & 19 & 6,5 & 9 & 47,4 & 10 & 52,6 & 2 & 10,5 & 17 & 89,5 \\
\hline P "mitrale" & 18 & 6,2 & 11 & 61,1 & 7 & 38,9 & 3 & 16,7 & 15 & 83,3 \\
\hline Sinus arrest & 15 & 5,1 & 3 & 20 & 12 & 80 & 4 & 26,7 & 11 & 73,3 \\
\hline $\begin{array}{l}\text { Bloqueio de ramo } \\
\text { direito ou sobrecarga } \\
\text { ventricular direita }\end{array}$ & 12 & 4,1 & 4 & 33,3 & 8 & 66,7 & 3 & 25 & 9 & 75 \\
\hline $\begin{array}{l}\text { Infradesnivelamento } \\
\text { de ST }\end{array}$ & 8 & 2,74 & 3 & 37,5 & 5 & 62,5 & 2 & 25 & 6 & 75 \\
\hline $\mathrm{BAV}$ de $1^{\circ}$ grau & 6 & 2,0 & 1 & 16,2 & 5 & 83,3 & - & - & 6 & 100 \\
\hline CVP & 6 & 2,0 & 4 & 66,7 & 2 & 33,3 & 1 & 16,7 & 5 & 83,3 \\
\hline Taquicardia sinusal & 3 & 1,0 & 2 & 66,7 & 1 & 33,3 & - & - & 3 & 100 \\
\hline $\begin{array}{l}\text { Supradesnivelamento } \\
\text { de ST }\end{array}$ & 3 & 1,0 & 1 & 33,3 & 2 & 66,7 & 1 & 33,3 & 2 & 66,7 \\
\hline $\begin{array}{l}\text { Taquicardia } \\
\text { ventricular }\end{array}$ & 3 & 1,0 & 2 & 66,7 & 1 & 33,3 & 1 & 33,3 & 2 & 66,7 \\
\hline Taquicardia atrial & 3 & 1,0 & 1 & 33,3 & 2 & 66,7 & - & - & 3 & 100 \\
\hline CAP & 3 & 1,0 & 2 & 66,7 & 1 & 33,3 & - & - & 3 & 100 \\
\hline P "pulmonale" & 2 & 0,7 & 1 & 50 & 1 & 50 & - & - & 2 & 100 \\
\hline Bradicardia sinusal & 2 & 0,7 & 2 & 100 & - & - & - & - & 2 & 100 \\
\hline Fibrilação atrial & 1 & 0,3 & 1 & 100 & - & - & - & - & 1 & 100 \\
\hline Ritmo idioventricular & 1 & 0,3 & 1 & 100 & - & - & - & - & 1 & 100 \\
\hline $\begin{array}{l}\text { Prolongamento do } \\
\text { "QT" }\end{array}$ & 1 & 0,3 & 1 & 100 & - & - & - & - & 1 & 100 \\
\hline $\begin{array}{l}\text { Complexo de escape } \\
\text { ventricular }\end{array}$ & 1 & 0,3 & 1 & 100 & - & - & - & - & 1 & 100 \\
\hline $\begin{array}{l}\text { Complexo de escape } \\
\text { juncional }\end{array}$ & 1 & 0,3 & 1 & 100 & - & - & - & - & 1 & 100 \\
\hline Taquicardia juncional & 1 & 0,3 & 1 & 100 & - & - & - & - & 1 & 100 \\
\hline $\begin{array}{l}\text { Complexo prematuro } \\
\text { juncional }\end{array}$ & 1 & 0,3 & 1 & 100 & - & - & - & - & 1 & 100 \\
\hline $\mathrm{BAV}$ de $2^{\circ}$ grau & 1 & 0,3 & 1 & 100 & - & - & - & - & 1 & 100 \\
\hline Total & 292 & 100 & 125 & & 167 & & 57 & & 235 & \\
\hline
\end{tabular}

*Alguns animais apresentaram mais de uma alteração. 


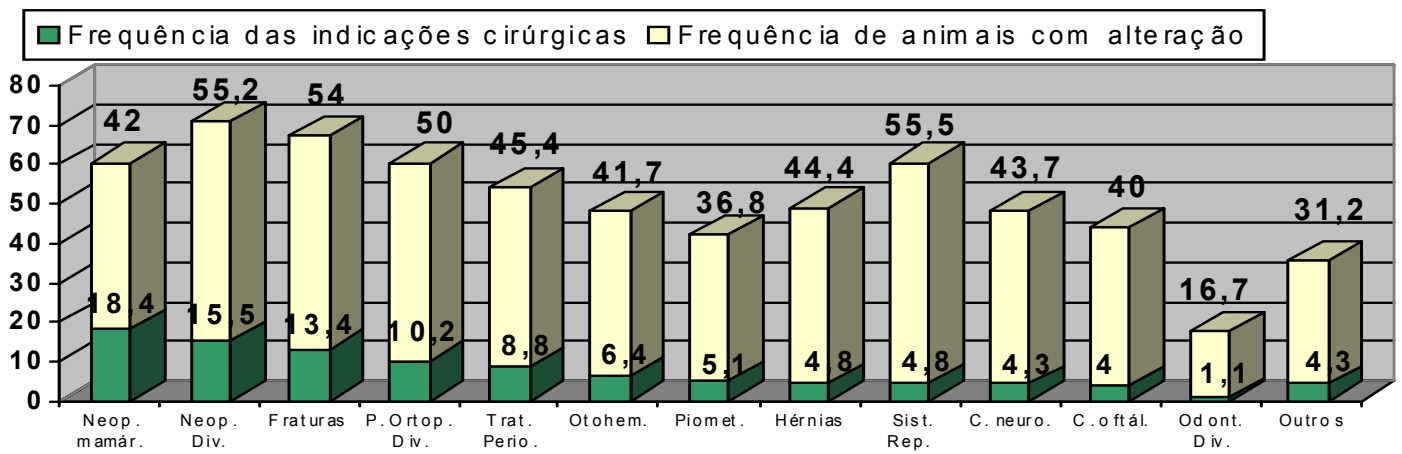

Figura 1. Frequência (\%) de indicações cirúrgicas em 374 cães atendidos no período de 03/2005 a 12/2006, de acordo com as alterações eletrocardiográficas pré-operatórias.

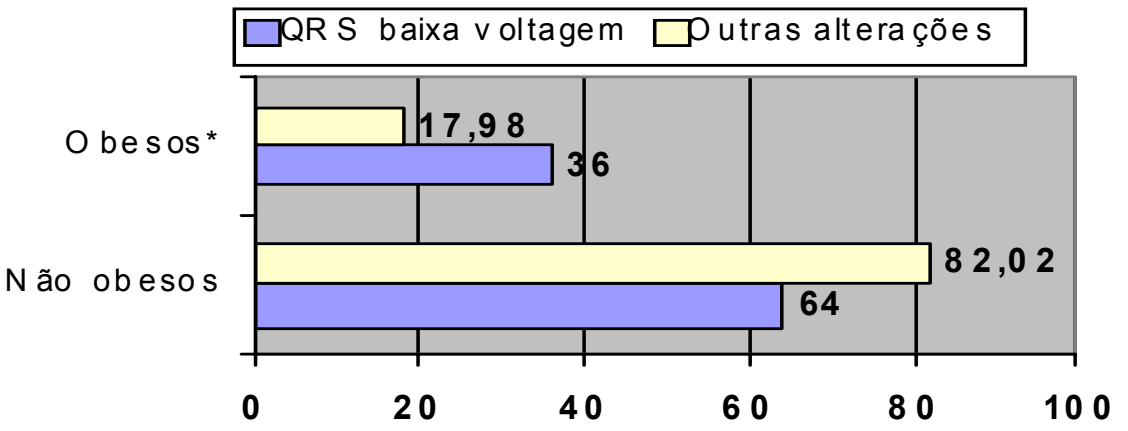

Figura 2. Frequência do complexo QRS de baixa voltagem e demais alterações observadas em 44 cães obesos e em 175 não obesos encaminhados para avaliação eletrocardiográfica pré-operatória no período de $03 / 2005$ a $12 / 2006$.

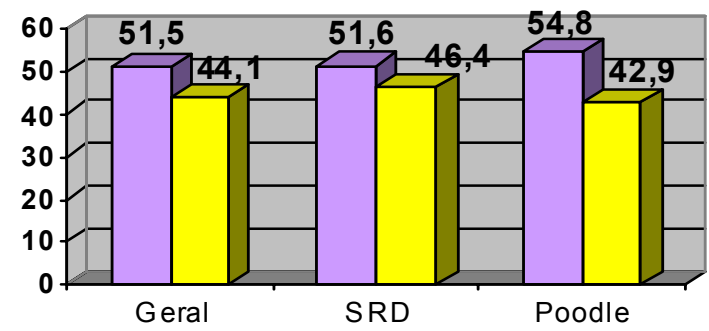

$\square$ Com Sinal Físico

$\square$ Sem Sinal Físico

Figura 3. Percentual de cães com alterações eletrocardiográficas pré-operatórias com e sem sinais físicos de doença cardíaca na população em geral e em cães SRD e Poodle, no período de 03/2005 a 12/2006.

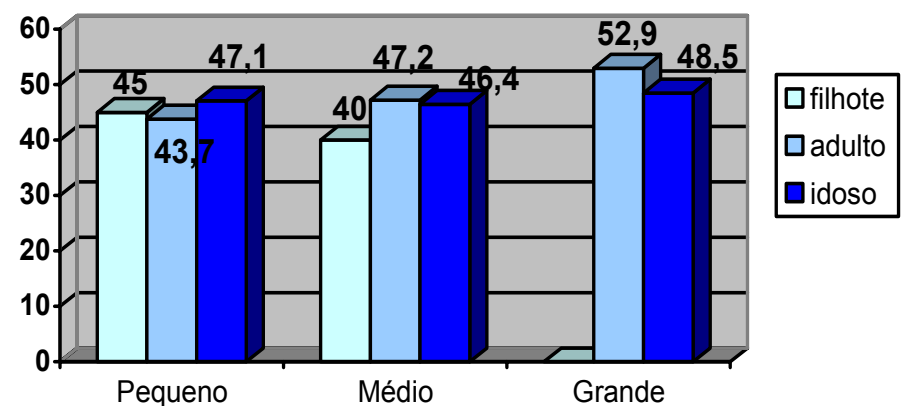

Figura 4. Percentual de alterações eletrocardiográficas no exame pré-operatório de cães de acordo com a idade e porte na população geral, no período de 03/2005 a 12/2006. 


\section{CONCLUSÕES}

A eletrocardiografia no pré-operatório de cães é um exame de grande importância, devendo ser realizada independente da enfermidade cirúrgica, em animais de todas as idades e pesos, que apresentem ou não histórico ou sinais físicos de alterações cardiovasculares e, em especial, nos pacientes obesos e do sexo masculino.

O autor I.V. Neves é bolsista de Iniciação Científica - UFRPE.

\section{REFERÊNCIAS BIBLIOGRÁFICAS}

ABBOTT, J.A. Doença valvular adquirida. In: TILLEY, L.P.; GOODWIN, J.K. Manual de cardiologia para cães e gatos. 3.ed. São Paulo: Roca, 2002. p.109-132.

ANYUKHOVSKY, E.P.; SOSUNOV, E.A.; PLOTNIKOV, A. et al. Cellular electrophysiologic properties of old canine atria provide a substrate for arrhythmogenesis. Cardiovasc. Res., v.54, p.462-469, 2002.

BROURMAN, J.D.; SCHERTEL, E.R.; ALLEN, D.A. et al. Factors associated with perioperative mortality in dogs with surgically mananged gastric dilatation-volvulus: 137 cases. J. Am. Vet. Med. Assoc., v.208, p.1855-1858, 1996.

CALVERT, C.A. Cardiomiopatia canina. In: TILLEY, L.P.; GOODWIN, J.K. Manual de cardiologia para cães e gatos. 3. ed. São Paulo: Roca, 2002. p.133-152.

CARR, A.P.; TILLEY, L.P.; MILLER, M.S. Tratamento de arritmias cardíacas e distúrbios de condução. In: TILLEY, L.P.; GOODWIN, J.K. Manual de cardiologia para cães e gatos. 3.ed. São Paulo: Roca, 2002. p.347-376.

DUERR, F.M.; CARR, A.P.; DUKE, T. et al. Prevalence of perioperative arrhythmias in 50 young, healthy dogs. Can. Vet. J., v.48, p.169177, 2007

EDWARDS, N.J. Bolton's handbook of canine and feline electrocardiography. 2.ed. Philadelphia: W.B. Saunders, 1987. p.15.

FERREIRA, W.L.; AYLON, E.G.; CARREGARO, A.B. Ação antiarrítmica do isofluorano em cães submetidos a arritmias ventriculares induzidas por cloreto de bário Arq.
Bras. Med. Vet. Zootec., v.58, p.1064-1069, 2006.

FRIES, C.L. Assessment and preparation of the surgical patient. In: SLATTER, D. Textbook of small animal surgery. 2.ed. Philadelphia: W.B. Saunders, 1993. p.137-140

FUTEMA, F. Avaliação pré-anestésica. In: FANTONI, D.T.; CORTOPASSI, S.R.G. Anestesia em cães e gatos. São Paulo: Roca, 2002. p.59-63.

GOMPF, R.E. A história e o exame físico. In: GOODWIN, J.K.; TILLEY, L.P. Manual de cardiologia para cães e gatos. 3.ed. São Paulo: Roca, 2002. p.3-14.

GOODWIN, J.K. Eletrocardiografia. In: GOODWIN, J.K.; TILLEY, L.P. Manual de cardiologia para cães e gatos. 3.ed. São Paulo: Roca, 2002. p.39-65.

GRANDJEAN, D. Enciclopédia do cão royal canin. Paris: Aniwa Publishing, 2001. 562p.

JERICÓ, M.M.; SILVA, M.B.F.P.; MACHADO, F.L.A., Avaliação cardiovascular em cães obesos: mensuração da pressão arterial e achados eletrocardiográficos. Clin. Vet., v.61, p.66-72, 2006.

KELLY, D.F. Interpretação eletrocardiográfica. In: DARKE, P.; BONAGURA, J.D.; KELLY, D.F. Atlas ilustrado de cardiologia veterinária. São Paulo: Manole, 2000. p.64-75.

KITTLESON, M.D. Signal, history and physical examination. In: KITTLESON, M.D.; KIENLE, R.D. Small animal cardiovascular medicine. St. Louis: Mosby, 1998. p.36-46.

LUNNEY, J.; ETTINGER, S. Arritmias cardíacas. In: ETTINGER, S.J.; FELDMAN, E.C. Tratado de medicina interna veterinária. 4.ed. São Paulo: Manole, 1997. p.1338-1381.

NELSON, R.W.; COUTO, C.G. Medicina interna de pequenos animais. Rio de Janeiro: Guanabara Koogan, 2001. 1084p.

ORTON, C.E. Cirurgia do sistema cardiovascular. In: FOSSUM, T.W. Cirurgia de pequenos animais. São Paulo: Roca, 2002. p.638-645.

PADDLEFORD, R.R. Drogas anestésicas In: PADDLEFORD, R.R. Manual de anestesia em 
pequenos animais. 2.ed. São Paulo: Roca, 2001. p.15-88.

RABELO, C.R. A importância da avaliação eletrocardiográfica como exame pré-operatório em cães. Belo Horizonte: Anclivepa-MG, 2004.

RAMIREZ, E.Y.; PALANCA, I.M.; PABLOBLANCO, J.B. et al. Arritmias cardíacas no cão e gato. In: BELERENIAN, G.C.; MUCHA, C.J.; CAMACHO, A.A. Afecções cardiovasculares em pequenos animais. São Paulo: Interbook, 2003. p.230-259.

ROGERS, M.C.: TINKER, J.H.; COVINA, B.G. et al. Princípios e prática de anestesiologia. Rio de Janeiro: Guanabara Koogan, 1993. p.54-62.

SISSON, D.; O'GRADY, M.R.; CALVERT, C.A. Myocardial diseases of dogs. In: FOX, P.R.; SISSON, D.D.; MÖISE, N.S. Textbook of canine and feline cardiology: principles and clinical practice. 2.ed. Philadelphia: W.B. Saunders, 1999. p.581-620.

SNYDER, P.S.; COOKE, K.L.; MURPHY, S.T. et al. Eletrocardiographic findings in dogs with motor vehicle - related trauma. J. Am. Anim. Hosp. Assoc., v.37, p.55-62, 2001.

TILLEY, L.P. Essential of canine and feline electrocardiography. 3.ed. Philadelphia: Lea \& Febiger, 1992. 470p.

TUDURY, E.A.; CAMACHO, A.A.; LAGÊDO, C.M.G. et al. Diagnóstico e tratamento das arritmias de cães e gatos, observadas no monitor cardíaco. Rev. CFMV, v.30, p.25-38, 2003.

WOLF, R.; CAMACHO, A.A.; SOUZA, R.A.A. Eletrocardiografia computadorizada em cães. Arq. Bras. Med. Vet. Zootec., v.52, p.610-615, 2000. 